PROCEEDINGS OF THE

AMERICAN MATHEMATICAL SOCIETY

Volume 135, Number 12, December 2007, Pages 4045-4055

S 0002-9939(07)08900-9

Article electronically published on August 15, 2007

\title{
PERIODIC HOMOTOPY AND CONJUGACY IDEMPOTENTS
}

\author{
JAKA SMREKAR
}

(Communicated by Alexander N. Dranishnikov)

\begin{abstract}
A self-map $f$ on the $\mathrm{CW}$ complex $Z$ is a periodic homotopy idempotent if for some $r \geqslant 0$ and $p>0$ the iterates $f^{r}$ and $f^{r+p}$ are homotopic. Geoghegan and Nicas defined the rotation index $R I(f)$ of such a map. They proved that for $r=p=1$, the homotopy idempotent $f$ splits if and only if $R I(f)=1$, while for $r=0$, the index $R I(f)$ divides $p^{2}$. We extend this to arbitrary $p$ and $r$, and generalize various results related to the splitting of homotopy idempotents on CW complexes and conjugacy idempotents on groups.
\end{abstract}

\section{Introduction. Periodic homotopy idempotents}

Let $p$ be a natural number. In any category, it makes sense to call the morphism $f: Z \rightarrow Z$ a $p$-idempotent if the iterate $f^{p+1}$ is equal to $f$. There is a distinguished class of $p$-idempotents $Z \rightarrow Z$, namely those that factor as $f=u \circ d$ for some morphisms $d: Z \rightarrow Y$ and $u: Y \rightarrow Z$ satisfying $(d u)^{p}=\mathrm{id}_{Y}$. These are called split $p$-idempotents, and any factorization $f=u d$ with this property is called splitting. Note that the isomorphism class of $Y$ is unique in this case. When emphasis is required, we call 1-idempotents ordinary idempotents. Note that if $f$ is a $p$-idempotent, then $f$ splits if and only if $f=u d$ with $d u$ an isomorphism.

The categories we are interested in here are those of groups (with morphisms conjugacy classes of group homomorphisms) and of CW complexes (with morphisms free homotopy classes of continuous maps).

If $f: Z \rightarrow Z$ is an ordinary homotopy idempotent on the connected CW complex $Z$ (this is to say $f^{2}$ is freely homotopic to $f$ ), then the induced morphism on the fundamental group $\phi=f_{\#}: \pi_{1}(Z) \rightarrow \pi_{1}(Z)$ is an ordinary conjugacy idempotent (i.e. $\phi^{2}$ is conjugate to $\phi$ ). Freyd and Heller (4], Main Lemma) have shown that $f$ splits up to homotopy if and only if $\phi$ splits up to conjugacy. Moreover, this happens if and only if the kernel of a certain universal morphism $\kappa_{\phi}: F \rightarrow \pi_{1}(Z)$ from the Thompson group $F$ has nontrivial kernel (see Brown and Geoghegan [3]).

Received by the editors April 26, 2006 and in revised form, September 6, 2006.

2000 Mathematics Subject Classification. Primary 55P99; Secondary 20F38, 57M07, 57M10.

Key words and phrases. Periodic homotopy idempotent, split idempotent, rotation index, eventual coherence, Thompson groups.

The author was supported in part by the MŠZŠ of the Republic of Slovenia research program No. P1-0292-0101-04 and research project No. J1-6128-0101-04, and in part by the DURSI of the Generalitat de Catalunya grant 2004-CRED-00048. 
In 6] Geoghegan and Nicas have given another necessary and sufficient condition for an ordinary homotopy idempotent to split, in relation with their one-parameter fixed point theory on mapping tori (see [5], §6). We need a definition.

Let $I=[0,1]$. For a self-map $f$ on the topological space $Z$, its mapping torus $T_{f}$ is the quotient space of $Z \times I$ modulo the relations $(z, 1) \sim(f(z), 0)$. It admits a projection $p_{f}: T_{f} \rightarrow S^{1}$ defined by $[z, t] \mapsto e^{2 \pi i t}$. Let $T_{f}^{T_{f}}$ denote the space of selfmaps $T_{f} \rightarrow T_{f}$ with the compact-open topology. Pick a base-point $z_{0}$ in $Z$ and set $\zeta_{0}=\left[z_{0}, 0\right]$. Let eval: $\left(T_{f}\right)^{T_{f}} \rightarrow T_{f}$ denote evaluation at $\zeta_{0}$. Denote the composite $P_{f}=p_{f}$ eval: $\left(T_{f}\right)^{T_{f}} \rightarrow S^{1}$. Set $\Gamma_{f}=\pi_{1}\left(\left(T_{f}\right)^{T_{f}}\right.$,id $)$. Identifying $\pi_{1}\left(S^{1}, 1\right)$ with the integers $\mathbb{Z}$ via degree, $P_{f}$ induces a morphism $P_{f \#}: \Gamma_{f} \rightarrow \mathbb{Z}$. Each element of $\Gamma$ may be represented (when the topology on $Z$ is reasonable) with a cyclic homotopy $h: T_{f} \times I \rightarrow T_{f}$, i.e., one beginning and ending in the identity. Evaluation then corresponds to taking the trace of the homotopy, i.e., the path $t \mapsto h\left(\zeta_{0}, t\right)$. The rotation index (Geoghegan and Nicas, [5]) $R I(f)$ of the self-map $f$ is the least positive integer in the image of $P_{f \#}$. If $P_{f \#}$ is trivial, we set $R I(f)=\infty$.

Geoghegan and Nicas have shown that an ordinary homotopy idempotent $f: Z \rightarrow$ $Z$ on the connected CW complex $Z$ splits if and only if $R I(f)=1$. An obvious problem is to find a characterization of $R I(f)=q$ for any $q$. If for some $r \geqslant 0$ and $p>0$ the iterates $f^{r}$ and $f^{r+p}$ are homotopic, the self-map $f$ is called a periodic homotopy idempotent. On nice spaces (e.g., compacta), $R I(f)=q$ implies $f^{i} \simeq f^{i+q}$ for some $i$. ( $\simeq$ denotes the 'free' homotopy relation.) Thus the interesting problems are the meaning of $R I(f)=q$ for $f^{r} \simeq f^{r+p}$, and also the relation between $R I(f)$ and $R I\left(f^{r}\right)$ in this case. Our main results are the following solutions:

Theorem 1.1. Let $Z$ be a connected space of $C W$ homotopy type and let $f$ be a self-map on $Z$ with $f^{r}$ freely homotopic to $f^{r+p}$. Then $R I(f)<\infty$ if and only if $f^{r}$ splits as a homotopy $p$-idempotent. In that case $R I(f)$ divides $p^{2}$.

Corollary 1.2. Let $f: Z \rightarrow Z$ be a periodic homotopy idempotent, $f^{r} \simeq f^{r+p}$. If for some $j \geqslant 1$ we have $R I\left(f^{j}\right)<\infty$ it follows that $R I\left(f^{i}\right)<\infty, \forall i \geqslant 1$. Moreover, this is true if and only if on the fundamental group $\operatorname{im} f_{\#}^{r}=\operatorname{im} f_{\#}^{r+1}=\cdots$.

Theorem 1.1 generalizes Theorems 2.4 and 3.4 of Geoghegan and Nicas [6] and in conjunction with Corollary 3.3 answers all questions of [6], Introduction. (See also [5], $§ 6$, page 35.) Moreover, in Theorem 4.3 below we characterize a finite rotation index by means of a universal morphism from a generalized Thompson group.

Theorem 1.1 depends on the investigation of split $p$-idempotents in section 2, In section 3 we list the basic properties of the rotation index, particulary in relation to eventual coherence where we point out an error in [5]. In section 4 we focus on periodic conjugacy idempotents. Section 5 contains the lengthier proofs.

\section{Split homotopy AND CONJUGACY $p$-IDEMPOTENTS}

As for $p=1$ (Freyd and Heller 4, Main Lemma), the question of splitting homotopy $p$-idempotents on $\mathrm{CW}$ complexes can be reduced to groups.

Theorem 2.1. Suppose $f$ is a base-point preserving self-map on the $C W$ complex $Z$, and that $f^{p+1}$ is freely homotopic to $f$. Then $f$ splits up to homotopy if and only if $f_{\#}: \pi_{1}(Z) \rightarrow \pi_{1}(Z)$ splits up to conjugacy.

Thus we turn to groups and consider an endomorphism $f: G \rightarrow G$ on the group $G$. For $\alpha$ in $G$ we let $f^{\alpha}$ denote the conjugate of $f$ by $\alpha$. Also, for elements $\xi$ and $\eta$ 
of $G$ we denote $\xi^{\eta}=\eta^{-1} \xi \eta$, so that $f^{\alpha}(\xi)=(f(\xi))^{\alpha}$. We denote the commutator $[\xi, \eta]=\xi \eta \xi^{-1} \eta^{-1}$. Recall the generalized Thompson group $F_{p}$ defined as

$$
F_{p}=\left\langle x_{0}, x_{1}, x_{2}, \ldots \mid x_{i}^{-1} x_{j} x_{i}=x_{j+p}, i<j\right\rangle .
$$

It admits a shift $\phi: F_{p} \rightarrow F_{p}$, defined by $\phi\left(x_{i}\right)=x_{i+1}$. The defining relations imply $\phi^{p+1}=\phi^{x_{0}}$ which is to say that $\phi$ is a conjugacy $p$-idempotent.

Lemma 2.2. Suppose $f: G \rightarrow G$ has $f^{p+1}=f^{\alpha}$. Then there exists a unique homomorphism $\kappa=\kappa_{f}: F_{p} \rightarrow G$ such that $\kappa\left(x_{0}\right)=\alpha$ and $\kappa \phi=f \kappa$.

This means that as for $p=1$, the $p$-idempotent $\phi$ is universal (cf. Brown and Geoghegan 3], Proposition 1.1). Moreover, as for $p=1$ (cf. Freyd and Heller [4], Main Theorem), $\phi$ is the universal nonsplitting $p$-idempotent. To wit, we prove

Theorem 2.3. Let $f: G \rightarrow G$ satisfy $f^{p+1}=f^{\alpha}$. The following are equivalent:

(i) $f$ splits.

(ii) The images of $f$ and $f^{2}$ coincide.

(iii) $[\alpha, f(\alpha)]=1$.

(iv) The kernel of the universal morphism $\kappa_{f}: F_{p} \rightarrow G$ is nontrivial.

(v) The kernel of $\kappa_{f}: F_{p} \rightarrow G$ contains the commutator subgroup.

The case $p=1$ is somewhat special in that there are critical points in the slick arguments of Freyd and Heller [4] that do not carry over to the case $p>1$. So Theorems 2.1 and 2.3 are proven here using a different, geometric, approach.

Corollary 2.4. (i) Let $f$ be either a homotopy or a conjugacy p-idempotent. Then $f$ splits if and only if $f^{p}$ splits as an idempotent.

(ii) Every homotopy p-idempotent on a finite-dimensional CW complex splits.

Proof. For (i) it suffices to consider groups, by virtue of Theorem 2.1. Let $f: G \rightarrow G$ satisfy $f^{p+1}=f^{\alpha}$. We note $\left(f^{p}\right)^{2}=f^{p+1} f^{p-1}=f^{\alpha} f^{p-1}=\left(f^{p}\right)^{\alpha}$. If $f^{p}$ splits as an idempotent, then $\left[\alpha, f^{p}(\alpha)\right]=1$. Hence $\kappa_{f}: F_{p} \rightarrow G$ has nontrivial kernel (because $x_{0} x_{p} x_{2 p}^{-1} x_{0}^{-1}$ is the normal form for $\left[x_{0}, x_{p}\right]$; see Lemma 5.5 below), and $f$ splits.

As every ordinary homotopy idempotent on a finite dimensional $\mathrm{CW}$ complex splits (cf. Hastings and Heller [7]), (ii) follows from (i).

\section{Properties of the rotation index. Eventual Coherence}

The rotation index does not depend on the choice of base-point $z_{0}$ in $Z$; see [6], Proposition 1.2. We recall the basic result on homotopy invariance.

Proposition 3.1 (Geoghegan and Nicas [6], Proposition 1.6). Let $h: Z \rightarrow Z^{\prime}$ be a homotopy equivalence, and let $f: Z \rightarrow Z$ and $f^{\prime}: Z^{\prime} \rightarrow Z^{\prime}$ be self-maps for which the composites $h \circ f$ and $f^{\prime} \circ h$ are homotopic. Then $R I(f)=R I\left(f^{\prime}\right)$.

Let $W$ be a path-connected space that is homotopy equivalent to the $\mathrm{CW}$ complex $Z$, and let $g$ be a self-map on $W$. By Proposition 3.1, $R I(g)=R I\left(g^{\prime}\right)$ for an obvious self-map $g^{\prime}$ on $Z$. If $z_{0} \in Z$ is any point, then $g^{\prime}$ is homotopic to some $f$ with $f\left(z_{0}\right)=z_{0}$. Again, $R I\left(g^{\prime}\right)=R I(f)$. Thus for our purposes it suffices to focus on base-point preserving self-maps on CW complexes.

The notion of eventual coherence of periodic homotopy idempotents has been introduced by Geoghegan and Nicas (see [5], Section 6, as well as [6]). 
Definition. A self-map $f$ on the path-connected space $Z$ is an eventually coherent periodic homotopy idempotent if, for some $i \geqslant 0$ and $q>0$, there is a homotopy $N: Z \times[0,1] \rightarrow Z$ between $f^{i}$ and $f^{i+q}$ such that $f N$ is homotopic to $N\left(f\left({ }_{-}\right),{ }_{-}\right)$ via a homotopy $Z \times[0,1] \times[0,1] \rightarrow Z$ that is constant on $Z \times\{0,1\}$.

The period of $f$ is the least $q$ for which there exists an $i$ with the above property. If such an $i$ does not exist, we let the period be infinite in accordance with inf $\emptyset=\infty$.

The following proposition is the content of [5], Theorem 6.3.

Proposition 3.2 (Geoghegan and Nicas [6]). Let $f$ be a self-map on the connected space $Z$.

(i) If $Z$ is compact, then the period of $f$ equals its rotation index $R I(f)$.

(ii) In general, if $f$ has period $q<\infty$, then $R I(f)$ is finite and divides $q$.

Corollary 3.3. If $Z$ is a compact space of $C W$ type, then every periodic homotopy idempotent on $Z$ is eventually coherent.

Proof. If $f^{r} \simeq f^{r+p}$, then $f^{r}$ splits by (ii) of Corollary 2.4. By Theorem 1.1, $R I(f)$ divides $p^{2}$. Proposition 3.2 renders $f$ eventually coherent of period $R I(f)$.

Example. In [5], Theorem 6.3 (and [6], Proposition 1.5) the authors claim that for any space $Z$ and any self-map $f$ the period and rotation index coincide, although at some point their proof requires compactness. The following is a counterexample. The idea is that in general $R I(f)<\infty$ does not imply that the period is finite.

Let $n \geqslant 2$ and construct the Eilenberg-MacLane complex $K(\mathbb{Z}, n)$ with basepoint $\xi$. For $i \geqslant 1$ set $Z_{i}=K(\mathbb{Z}, n)^{p+i p}$ and define the self-map $f_{i}$ on $Z_{i}$ by

$$
f_{i}\left(x_{1}, \ldots, x_{p-1}, x_{p} ; y_{1}, \ldots, y_{i p-1}, y_{i p}\right)=\left(x_{2}, \ldots, x_{p}, x_{1} ; y_{2}, \ldots, y_{i p}, \xi\right) .
$$

Evidently $f_{i}^{i p+p}=f_{i}^{i p}$. On $\pi_{n} \cong \mathbb{Z}^{p+i p}$, the induced morphism $f_{i *}$ permutes the 'first' $p$ generators, and acts nilpotently with order $i p$ on the 'remaining' ip generators. Therefore clearly $f_{i}^{j_{1}} \simeq f_{i}^{j_{2}}$ for $j_{1}<j_{2}$ implies $j_{1} \geqslant i p$ and $j_{2}-j_{1}=k p$ for some positive $k$. In particular, the period of $f_{i}$ is $p$.

Since $f_{i}$ fixes the base-point $(\xi, \ldots, \xi)=\{\xi\}^{p+i p}$, the $f_{i}$ induce an obvious selfmap $f$ on $Z=\bigvee_{i=1}^{\infty} Z_{i}$. Note that $f$ cannot be a periodic homotopy idempotent.

Let $\hat{f}_{i}$ denote the induced map on the mapping torus $T_{f_{i}}$. Evidently $\hat{f}_{i}^{p+i p}=\hat{f}_{i}^{i p}$. The concatenation $R_{p+i p} * R_{i p}^{-1}$ of the corresponding 'tumbles' (see the notes before Lemma 5.6) is a cyclic homotopy $\bar{h}_{i}: T_{f_{i}} \times I \rightarrow T_{f_{i}}$ of rotation degree $p$ (see also the discussion in [5] preceding Theorem 6.3). We want to glue these to get a cyclic homotopy $h: T_{f} \times I \rightarrow T_{f}$ of degree $p$. The torus $T_{f}$ is the 'union' (=colimit) of the tori $T_{f_{i}}$ meeting in the common circle $S^{1}=\{[*, t] \mid t \in[0,1]\}$ where $*$ is the generic base-point. The $\bar{h}_{i}$ do not agree along $S^{1}$. However, for each $i$ the restriction $\left.\bar{h}_{i}\right|_{S^{1} \times I}$ is a map $S^{1} \times I \rightarrow S^{1}$ representing an element in $\pi_{1}\left(\left(S^{1}\right)^{S^{1}}\right.$,id) of degree $p$. Fix a representative for that map. Each $\bar{h}_{i}$ is homotopic as a cyclic homotopy to some $h_{i}$ extending the fixed map $S^{1} \times I \rightarrow S^{1}$. The $h_{i}$ together define a cyclic homotopy $T_{f} \times I \rightarrow T_{f}$ of degree $p$. The self-map $f$ is not a periodic homotopy idempotent but has $R I(f)=p$, contradicting Theorem 6.3 of [5].

It seems hard to get a grip on the period for noncompact spaces. The rotation index is easier to deal with and adequate for comparison with splitting phenomena. 


\section{Periodic COnJugacy idempotents}

We consider the following generalization of the Thompson group $F_{p}$ :

$$
F_{p, r}=\left\langle x_{0}, x_{1}, x_{2}, \ldots \mid x_{i}^{-1} x_{j} x_{i}=x_{j+p}, \quad i+r \leqslant j\right\rangle .
$$

Also $F_{p, r}$ admits the shift $\phi\left(x_{i}\right)=x_{i+1}$, and evidently $\phi^{r+p}=\left(\phi^{r}\right)^{x_{0}}$, i.e., $\phi$ is a periodic conjugacy $p$-idempotent. Note that $F_{p}=F_{p, 1}$ is a quotient of $F_{p, r}$. As for $p$-idempotents, $\phi: F_{p, r} \rightarrow F_{p, r}$ is universal in the following sense.

Lemma 4.1. Suppose $f: G \rightarrow G$ has $f^{p+r}=\left(f^{r}\right)^{\alpha}$. Then there exists a unique homomorphism $\kappa=\kappa_{f}: F_{p, r} \rightarrow G$ such that $\kappa\left(x_{0}\right)=\alpha$ and $\kappa \phi=f \kappa$.

As $\phi^{r+p}=\left(\phi^{r}\right)^{x_{0}}$, it follows that $\left(\phi^{r}\right)^{1+p}=\phi^{r+r p}=\left(\phi^{r}\right)^{x_{0}^{r}}$, that is, $\phi^{r}$ is a $p$-idempotent. Denote the shift $\psi: F_{p} \rightarrow F_{p}$. The morphism $\kappa_{p, r}: F_{p} \rightarrow F_{p, r}$ with $\kappa_{p, r}\left(x_{0}\right)=x_{0}^{r}$ and $\kappa_{p, r} \psi=\phi^{r} \kappa_{p, r}$ of Lemma 2.2 is given by $\kappa_{p, r}\left(x_{i}\right)=x_{r i}^{r}$. Denote the quotient morphism $q_{p, r}: F_{p, r} \rightarrow F_{p}$.

Proposition 4.2. The composite $F_{p} \stackrel{\kappa_{p, r}}{\longrightarrow} F_{p, r} \stackrel{q_{p, r}}{\longrightarrow} F_{p}$ is injective. Consequently $\kappa_{p, r}$ is injective, $\phi^{r}: F_{p, r} \rightarrow F_{p, r}$ does not split, and $R I(\phi)=\infty$.

(For a morphism $\vartheta: G \rightarrow G$ we can define $R I(\vartheta)$ to be $R I(f)$ for any self-map on the Eilenberg-MacLane space $K(G, 1)$ inducing $\vartheta$ on $\pi_{1}$.)

We infer the following analogue of equivalence $(\mathbf{i}) \Longleftrightarrow($ iv $)$ of Theorem 2.3 .

Theorem 4.3. Let $G$ be a group and $f: G \rightarrow G$ a morphism with $f^{r+p}$ conjugate to $f^{r}$. Let $\kappa_{f}: F_{p, r} \rightarrow G$ be the associated morphism. Then $R I(f)<\infty$ if and only if the restriction of $\kappa_{f}$ to the image of $\kappa_{p, r}$ has nontrivial kernel.

Proof. Let $\kappa^{\prime}: F_{p} \rightarrow G$ be the morphism associated to the $p$-idempotent $f^{r}$. Then $\kappa^{\prime}=\kappa_{f} \circ \kappa_{p, r}$, and the assertion follows from Proposition 4.2 and Theorem 2.3 .

Example. The shift $\phi$ on $F_{p, r}$ has $\phi^{r+p}=\left(\phi^{r}\right)^{x_{0}}$; hence $f=\phi^{r}$ satisfies $f^{r+p}=$ $\left(f^{r}\right)^{\alpha}$ for $\alpha=x_{0}^{r}$. Corollary 1.2 implies that $R I(f)=\infty$. Let $\kappa_{f}: F_{p, r} \rightarrow F_{p, r}$ denote the universal morphism. As $f$ is also a $p$-idempotent, it has a universal morphism $F_{p} \rightarrow F_{p, r}$ in the sense of Lemma 2.2. It is precisely $\kappa_{p, r}$ which is injective. As $\kappa_{f}=\kappa_{p, r} q_{p, r}$, the kernel of $\kappa_{f}$ equals that of $q_{p, r}$, and is nontrivial.

\section{Proofs}

Let $f: Z \rightarrow Z$ be a self-map. The mapping telescope $\operatorname{Tel}_{f}$ of $f$ is the quotient space of the disjoint union $\bigsqcup_{n \in \mathbb{Z}} Z \times\{n\} \times[0,1]$ modulo the relations $(z, n, 1) \sim$ $(f(z), n+1,0)$. We use $[z, n, t] \in \operatorname{Tel}_{f}$ for the point represented by $(z, n, t)$. Another frequently used telescope is the subspace $\mathrm{Tel}_{f}^{+}$of $\mathrm{Tel}_{f}$ consisting of all points $[z, n, t]$ with $n \geqslant 0$. Note that $\mathrm{Tel}_{f}^{+}$is a strong deformation retract of $\mathrm{Tel}_{f}$.

We say that a continuous map $f: X \rightarrow Y$ factors strictly through the topological space $W$ if there exist $g: X \rightarrow W$ and $h: W \rightarrow Y$ such that $h \circ g=f$ pointwise.

For $f:\left(X, x_{0}\right) \rightarrow\left(Y, y_{0}\right)$ and generic $k \geqslant 1$ we denote the induced morphism on homotopy groups as $f_{*}: \pi_{k}\left(X, x_{0}\right) \rightarrow \pi_{k}\left(Y, y_{0}\right)$. When the fundamental group is emphasized, we use $f_{\#}: \pi_{1}\left(X, x_{0}\right) \rightarrow \pi_{1}\left(Y, y_{0}\right)$.

Our first goal is to prove Theorems 2.3 and 2.1. The first two auxiliary results will also be applied later on in the proof of Theorem 1.1 .

Lemma 5.1. Let $f: Z \rightarrow Z$ be a self-map. If for some $r \geqslant 0$ and $p \geqslant 1$ the iterates $f^{r}$ and $f^{r+p}$ are homotopic, then $f^{r}$ factors strictly through $\mathrm{Tel}_{f}^{+}$(and $\mathrm{Tel}_{f}$ ). 
Proof. Assume that $N: Z \times I \rightarrow Z$ is a homotopy between $f^{r}$ and $f^{r+p}$. Let $d: Z \rightarrow \operatorname{Tel}_{f}^{+}$be the inclusion $z \mapsto[z, 0,0]$ and define $u: \mathrm{Tel}_{f}^{+} \rightarrow Z$ by setting $u([z, n, t])=f^{n(p-1)}(N(z, t))$. Evidently $u$ is well defined and $u d=f^{r}$.

For $\mathrm{Tel}_{f}$ precompose $u$ with the retraction $\mathrm{Tel}_{f} \rightarrow \mathrm{Tel}_{f}^{+}$.

Proposition 5.2. Let $f:\left(Z, z_{0}\right) \rightarrow\left(Z, z_{0}\right)$ be a self-map on the pointed space of $C W$ type $\left(Z, z_{0}\right)$. Suppose that $f$ is homotopic to $f^{p+1}$ via homotopy $N: Z \times I \rightarrow Z$. Let $\nu \in \pi_{1}\left(Z, z_{0}\right)$ denote the class of the loop $t \mapsto N\left(z_{0}, t\right)$ traced out under $N$ by the base-point. Assume the factorization of $f^{r}$ through $\mathrm{Tel}_{f}^{+}$defined above. Let $z_{0}$ and $\left[z_{0}, 0,0\right]$ be the base-points in $Z$ and $\mathrm{Tel}_{f}^{+}$, respectively.

Assume that for each $k \geqslant 1$ and each $\gamma \in \pi_{k}\left(Z, z_{0}\right)$ there exist positive numbers $r_{0}$ and $q$ as well as an element $\eta \in \pi_{1}\left(Z, z_{0}\right)$ with the property that

$$
f_{*}^{r+\lambda q}(\gamma)=\left[f_{*}^{r}(\gamma)\right]_{\#}^{f_{\#}^{r+\lambda q}\left(\eta^{\lambda}\right)}
$$

for all $r \geqslant r_{0}$ and all $\lambda \geqslant 0$. Then for every $k \geqslant 1$ the restriction

$$
\left.d_{*}\right|_{\operatorname{im} f_{*}}: \operatorname{im} f_{*} \rightarrow \pi_{k}\left(\mathrm{Tel}_{f}^{+}\right)
$$

is an isomorphism and the factorization of $f$ through its telescope $\mathrm{Tel}_{f}^{+}$is splitting.

Proof. Let $G_{k}=\pi_{k}\left(Z, z_{0}\right)$. Then $\pi_{k}\left(\mathrm{Tel}_{f}^{+}, z_{0}\right)$ is isomorphic with the colimit $C_{k}$ of

$$
G_{k}^{0} \stackrel{f_{*}}{\longrightarrow} G_{k}^{1} \stackrel{f_{*}}{\longrightarrow} G_{k}^{2} \rightarrow \cdots,
$$

where $G_{k}^{i}=G_{k}$ for all $i$. The morphism $d_{*}: G_{k} \rightarrow C_{k}$ corresponds to inclusion on the first level, i.e., $G_{k} \equiv G_{k}^{0} \rightarrow C_{k}$. To determine its kernel suppose that $d_{*}(\gamma)=1$. This is true if and only if $f^{\mu}(\gamma)=1$ for all large enough $\mu$. Take $\mu=\lambda p+1$. Then

$$
f_{*}^{\mu}=f_{*}^{\lambda p+1}=f_{*}^{\nu^{\lambda}},
$$

and $f_{*}^{\mu}(\gamma)=\left(f_{*}(\gamma)\right)^{\nu^{\lambda}}$ is trivial if and only if $f_{*}(\gamma)$ is. Thus ker $d_{*}=\operatorname{ker} f_{*}$. The same argument shows that $f_{*}$, and hence $d_{*}$, are injective on the image of $f_{*}$.

Next we show that $d_{*}$ is surjective. Pick an element $\xi$ of $C_{k}$. It is represented by an element $\gamma \in G_{k}^{\mu^{\prime}}$ for some $\mu^{\prime}$. Let $r_{0}, q$, and $\eta$ be as in the statement of the proposition. There is an $r \geqslant r_{0}$ such that $\mu=\mu^{\prime}+r=\lambda q$ for some $\lambda$. Then $\xi$ is also represented by $f_{*}^{r}(\gamma) \in G_{k}^{\mu}$. We set $\Gamma=f_{*}^{r-1}\left(\gamma^{\eta^{-\lambda}}\right)$ and rewrite $(\dagger)$ as

$$
f_{*}^{r}(\gamma)=\left(f_{*}^{r+\lambda q}\right)^{f_{\#}^{r+\lambda q}\left(\eta^{-\lambda}\right)}(\gamma)=f_{*}^{r+\lambda q}\left(\gamma^{\eta^{-\lambda}}\right)=f_{*}^{\mu}\left(f_{*}(\Gamma)\right) .
$$

But $f_{*}^{\mu}\left(f_{*}(\Gamma)\right)$ in $G_{k}^{\mu}$ is in the image of $f_{*}(\Gamma) \in G_{0}^{k}$. This shows that the restriction of $d_{*}$ to the image of $f_{*}$ is surjective onto $C_{k}$. In particular, the morphism ( $\star$ ) is an isomorphism, as claimed.

The resulting short exact sequence $1 \rightarrow \operatorname{ker} d_{*} \rightarrow G_{k} \rightarrow C_{k} \rightarrow 1$ with ker $d_{*}=$ ker $f_{*}$ induces the isomorphism $h: \operatorname{im} f_{*} \rightarrow C_{k}$ defined by $h\left(f_{*}(x)\right)=d_{*}(x)$.

Thus $f_{*}=u_{*} d_{*}=u_{*} h f_{*}$ which is to say that $u_{*} h$ is the identity on $\operatorname{im} f_{*}$. Then $d_{*} u_{*} h: \operatorname{im} f_{*} \rightarrow C_{k}$ is an isomorphism. Since $h$ is an isomorphism, $d_{*} u_{*}: C_{k} \rightarrow C_{k}$ is also an isomorphism. By Whitehead's theorem, the composite $d u: \mathrm{Tel}_{f}^{+} \rightarrow \mathrm{Tel}_{f}^{+}$ is a homotopy equivalence, hence the factorization of $f$ is splitting.

Addendum. Assume the notation employed in the statement of the proposition. If $\nu$ lies in the image of $f_{\#}$, i.e., $\nu=f_{\#}(\eta)$, then $(\dagger)$ holds for this $\eta$ together with $r_{0}=1$ and $q=p$ for all $\gamma$. Indeed, recall first that $f_{*}^{\lambda p+1}=f_{*}^{\nu^{\lambda}}$. Using $\nu=f_{\#}(\eta)$, 
we compute $f_{\#}^{p+1}(\eta)=f_{\#}^{\nu}(\eta)=\nu^{-1} f_{\#}(\eta) \nu=f_{\#}(\eta)$. Thus $f_{\#}\left(\eta^{\lambda}\right)=f_{\#}^{\lambda p+1}\left(\eta^{\lambda}\right)$ for all $\lambda$. Substituting this into the equation $f_{*}^{\lambda p+1}=f_{*}^{f_{\#}\left(\eta^{\lambda}\right)}$ yields $(\dagger)$.

Realizing a group morphism as a map between Eilenberg-MacLane spaces we immediately deduce from Proposition 5.2 and the addendum the following.

Corollary 5.3. Let $g: G \rightarrow G$ be a group endomorphism which is a p-idempotent up to conjugation, $g^{p+1}=g^{\nu}$. If $\nu$ lies in the image of $g$, then $g$ splits.

Proposition 5.4. Suppose $g: G \rightarrow G$ is an endomorphism of groups which is a p-idempotent up to conjugation, $g^{p+1}=g^{\beta}$. If $[\beta, g(\beta)]=1$, then $h=g^{\beta}$ has $h^{p+1}=h^{h(\eta)}$ for some $\eta$ (and $\left.[\eta, h(\eta)]=1\right)$.

Proof. First we compute

$(*) \quad h^{p+1}=g^{p} g^{(p+1) p+1}=g^{p} g^{\beta g^{p}(\beta) g^{2 p}(\beta) \cdots g^{p p}(\beta)}=\left(g^{p+1}\right)^{g^{p}(\beta) g^{2 p}(\beta) \cdots g^{(p+1) p}(\beta)}$.

From $[\beta, g(\beta)]=1$ it follows that $g^{p+1}(\beta)=g^{\beta}(\beta)=[g(\beta)]^{\beta}=g(\beta)$. Thus $g^{k p+i}(\beta)=g^{i}(\beta)$ for all $i, k>0$ (and $\left.h^{i}(\beta)=g^{i}(\beta)\right)$. Using this in $(*)$ we express $h^{p+1}=\left(g^{p+1}\right)^{g^{(p+1) p}(\beta)}=h^{h^{p}(\beta)}$, which proves the assertion for $\eta=h^{p-1}(\beta)$.

Lemma 5.5 (Normal forms). Any $x \in F_{p}$ can be written in the form

$$
x=x_{i_{1}} \cdots x_{i_{k}} x_{j_{m}}^{-1} \cdots x_{j_{1}}^{-1} \quad \text { with } \quad i_{1} \leqslant \cdots \leqslant i_{k}, j_{1} \leqslant \cdots \leqslant j_{m}, k, m \geqslant 0 .
$$

This expression for $x$ can be chosen so that if $x_{i}$ and $x_{i}^{-1}$ both occur for some $i$, then $x_{j}$ or $x_{j}^{-1}$ also occurs for some $j \in i+1, \ldots, i+p$. Otherwise there would be a subproduct of the form $x_{i} \phi^{i+p+1}(y) x_{i}^{-1}$ which we can replace with $\phi^{i+1}(y)$. We call such an expression a normal form for $x$. Every $x \neq 1$ has a unique normal form.

Proof. See Remarks, (2), in Brown [2, page 58, and Brown and Geoghegan [3], 1.3, as well as the paragraph preceding Proposition 2.1.5 of Brin and Guzmán [1].

Proof of Proposition 4.2. Take $x \neq 1$ in $F_{p}$ and let $x=x_{i_{1}} \cdots x_{i_{k}} x_{j_{m}}^{-1} \cdots x_{j_{1}}^{-1}$ be the normal form for $x$ (see Lemma 5.5). Denote $\mu=q_{p, r} \circ \kappa_{p, r}: F_{p} \rightarrow F_{p}$. Then

$$
\mu(x)=x_{r i_{1}}^{r} \cdots x_{r i_{k}}^{r} x_{r j_{m}}^{-r} \cdots x_{r j_{1}}^{-r} .
$$

If $(\bullet)$ is a normal form, then $\mu(x) \neq 1$. Otherwise, there exists an $i$ such that in $(\bullet)$ there occur both $x_{r i}^{r}$ and $x_{r i}^{-r}$ but neither $x_{k}^{r}$ nor $x_{k}^{-r}$ for $k \in r i+1, \ldots, r i+p$. We may assume $i$ is maximal with this property.

Let $j$ be the smallest number bigger than $i$ such that one of $x_{j}$ and $x_{j}^{-1}$ occurs in $x$. By assumption, $r j-r i>p$. Divide $r j-r i=\lambda p+\delta$ where $\delta \in\{1, \ldots, p\}$. For all $k>r i$ we substitute $x_{k}^{ \pm r}$ in $(\bullet)$ for $x_{r i}^{-\lambda} x_{k-\lambda p}^{ \pm r} x_{r i}^{+\lambda}$, rendering the subexpression with indices $l \geqslant r i$ a normal form (after expansion of powers). As $j-i \leqslant p$, we have $r j-$ $r i \leqslant r p$ and thus $\lambda<r$. Thus in the subexpression in question we couldn't possibly eliminate the pair $x_{r i}$ and $x_{r i}^{-1}$. Moreover, the number of different generators and their inverses in $(\bullet)$ did not change after this transformation. Repeating this we get the normal form for $\mu(x)$ with the same number of different generators and their inverses as in $(\bullet)$ and hence as in the normal form for $x$. Thus $\mu(x) \neq 1$.

Proof of Theorem 2.3. Let $g: G \rightarrow G$ be a conjugacy $p$-idempotent, $g^{p+1}=g^{\beta}$.

To prove that (i) implies (ii), assume that $g$ splits. This is to say that $g$ is conjugate to $u d$, say $g=(u d)^{\alpha}$, with $d u$ an isomorphism. Then $g=u^{\alpha} d$ and 
$d u^{\alpha}=(d u)^{d(\alpha)}$ is an isomorphism. Thus $g^{2}=u^{\alpha} d u^{\alpha} d=u^{\alpha}\left(d u^{\alpha}\right) d$, and since $d u^{\alpha}$ is an isomorphism, the image of $g^{2}$ is the same as that of $g$.

For $(\mathbf{i i}) \Longrightarrow\left(\right.$ iii), assume im $g^{2}=\operatorname{im} g$. We have $g^{p+2}=g g^{p+1}=g g^{\beta}=\left(g^{2}\right)^{g(\beta)}$ and on the other hand, $g^{p+2}=g^{p+1} g=g^{\beta} g=\left(g^{2}\right)^{\beta}$. Thus the actions of $\beta$ and $g(\beta)$ by conjugation on im $g^{2}=\operatorname{im} g$ coincide. In particular, $\beta$ and $g(\beta)$ act in the same way on $g(\beta)$, i.e., they commute.

Evidently (iii) implies (i) by Proposition 5.4 and Corollary 5.3 .

The existence of normal forms by Lemma 5.5 guarantees that (iii) implies (iv). By Proposition 2.1.5 of Brin and Guzmán 1] (our $F_{p}$ is $F_{p+1,0}^{*}$ there), (iv) implies (v). By definition, (v) implies (iii).

Proof of Theorem 2.1. Let $N: f \simeq f^{p+1}$ have trace $\alpha$. In particular $f_{\#}^{p+1}=f_{\# \text {. }}^{\alpha}$.

Suppose that $f_{\#}$ splits. Then by implication (i) $\Longrightarrow$ (iii) of Theorem 2.3 and Proposition 5.4 the map $h=f^{p+1} \simeq f$ admits a homotopy $M: h \simeq h^{p+1}$ with trace $h_{\#}(\eta)$, for some $\eta$. (E.g., we may take for $M$ the concatenation of homotopies $f^{p} N * f^{2 p} N * \cdots * f^{(p+1) p} N$.) By Proposition [5.2, $h$ splits. Hence so does $f$.

To prove Theorem 1.1 we will need a handful of auxiliary results. First we discuss the basic properties of mapping telescopes and mapping tori.

The group of integers $\mathbb{Z}$ acts on $\mathrm{Tel}_{f}$ by 'integer translations' $\tau_{k}: \mathrm{Tel}_{f} \rightarrow \mathrm{Tel}_{f}$, defined by $\tau_{k}([z, n, t])=[z, n+k, t]$. This is a properly discontinuous action with orbit space $T_{f}$, and the associated projection $\rho: \operatorname{Tel}_{f} \rightarrow T_{f},[z, n, t] \mapsto[z, t]$, is a regular covering with group of covering translations exactly $\left\{\tau_{k} \mid k \in \mathbb{Z}\right\}$.

For $k \geqslant 0$, the $k$-th tumble (see Geoghegan and Nicas [5], page 32) is the homotopy $R_{k}: T_{f} \times I \rightarrow T_{f}$, defined by $R_{k}([z, u], s)=\left[f^{\lfloor u+q s\rfloor}(z), \bmod (u+q s)\right]$ where $\lfloor x\rfloor$ denotes the lower integer part of $x$ and $\bmod (x)=x-\lfloor x\rfloor$ its modulus. The standard lifting of $R_{k}$ to the telescope is the homotopy $\bar{R}_{k}$ : $\operatorname{Tel}_{f} \times I \rightarrow \operatorname{Tel}_{f}$, given by $([z, n, u], s) \mapsto\left[f^{\lfloor u+q s\rfloor}(z), n+\lfloor u+s q\rfloor, \bmod (u+q s)\right]$. It is an equivariant homotopy between the identity and the composite $\tau_{k} \circ \bar{f}^{k}$. In particular, $\bar{f}$ is homotopic to $\tau_{-1}$ which is a homeomorphism. Thus $\bar{f}$ is always a homotopy equivalence.

We define an explicit map $\beta: \mathrm{Tel}_{f} \rightarrow \mathrm{Tel}_{f^{r}}$ by setting

$$
[z, r m, u] \mapsto[z, m, u] \text { and }[z, r m+i, u] \mapsto\left[f^{r-i}(z), m+1,0\right], \quad 1 \leqslant i \leqslant r-1 .
$$

Note that $\beta$ is well-defined and continuous and satisfies $\beta \circ \bar{f}=\bar{f} \circ \beta$ where the latter $\bar{f}$ denotes the self-map on $\mathrm{Tel}_{f^{r}}$ induced by $f$. Evidently $\beta \circ \tau_{r}=\tau_{1} \circ \beta$.

Next we define an explicit map $\alpha: \operatorname{Tel}_{f^{r}} \rightarrow \operatorname{Tel}_{f}$ by setting

$$
\alpha[z, n, u]=\left[f^{\lfloor r u\rfloor}(z), r n+\lfloor r u\rfloor, \quad \bmod (r u)\right] .
$$

It is evident upon inspection that $\alpha \circ \tau_{1}=\tau_{r} \circ \alpha$.

It is easy to see that $\alpha$ and $\beta$ are mutually inverse homotopy equivalences. In particular, the composite $\beta \alpha$ is equivariantly homotopic to the identity on $\mathrm{Tel}_{f^{r}}$.

Lemma 5.6. Let $f:\left(Z, z_{0}\right) \rightarrow\left(Z, z_{0}\right)$ be a self-map on the $C W$ complex $Z$ and $\bar{f}: \mathrm{Tel}_{f} \rightarrow \mathrm{Tel}_{f}$ the induced map. Furthermore, let $d: Z \rightarrow \mathrm{Tel}_{f}$ denote the standard inclusion. If for each $k$ there exists a subgroup $B_{k}$ of $\pi_{k}(Z)$ such that the restriction $\left.d_{*}\right|_{B_{k}}: B_{k} \rightarrow \pi_{k}\left(\mathrm{Tel}_{f}\right)$ is an isomorphism, then $R I(f)=R I(\bar{f})$.

Proof. First we define a map from the mapping torus of $f$ to that of $\bar{f}$,

$$
j: T_{f} \rightarrow T_{\bar{f}}, \quad[z, u] \mapsto[[z, 0,0], u] .
$$


Note that $j$ is well-defined and continuous and that it lifts to $\bar{j}: \operatorname{Tel}_{f} \rightarrow \operatorname{Tel}_{\bar{f}}$, $[z, n, u] \mapsto[[z, 0,0], n, u]$. Denote the projection $p_{\bar{f}}: T_{\bar{f}} \rightarrow S^{1}$. As $p_{\bar{f}} \circ j=p_{f}$, the map $j$ is a homotopy equivalence if and only if its lifting $\bar{j}$ is a homotopy equivalence.

Since $\bar{f}: \operatorname{Tel}_{f} \rightarrow \operatorname{Tel}_{f}$ is a homotopy equivalence, so is the standard inclusion $\bar{d}: \operatorname{Tel}_{f} \rightarrow \operatorname{Tel}_{\bar{f}},[z, n, u] \mapsto[[z, n, u], 0,0]$ of $\operatorname{Tel}_{f}$ into the telescope of $\bar{f}$. Also denoting the standard inclusion $d: Z \rightarrow \operatorname{Tel}_{f}$, defined by $z \mapsto[z, 0,0]$, we note that $\bar{j} \circ d=\bar{d} \circ d$. We get the induced equality $\bar{j}_{*} \circ d_{*}=\bar{d}_{*} \circ d_{*}$ on $\pi_{k}$, and consequently

$$
\bar{j}_{*} \circ d_{*}^{\prime}=\bar{d}_{*} \circ d_{*}^{\prime}
$$

where $d_{*}^{\prime}$ denotes the restriction of $d_{*}$ to $B_{k}$. By hypothesis, $d_{*}^{\prime}$ is an isomorphism $B_{k} \rightarrow \pi_{k}\left(\mathrm{Tel}_{f}\right)$. But $\bar{d}_{*}$ is also an isomorphism, and the above equation implies that so is $\bar{j}_{*}$. Thus $\bar{j}$ induces isomorphisms on the homotopy groups. By Whitehead's theorem it is a homotopy equivalence. Hence so is $j$.

As $j:\left(T_{f},\left[z_{0}, 0\right]\right) \rightarrow\left(T_{\bar{f}},\left[\left[z_{0}, 0,0\right], 0\right]\right)$ is a homotopy equivalence, it is a pointed homotopy equivalence with a pointed inverse $j^{\prime}$ and, in particular, a base-point preserving homotopy $h: T_{\bar{f}} \times I \rightarrow T_{\bar{f}}$ between the identity and $j j^{\prime}$. The map

$$
K: T_{f}^{T_{f}} \rightarrow T_{\bar{f}}^{T_{\bar{f}}}, \gamma \mapsto j \circ \gamma \circ j^{\prime},
$$

is also a homotopy equivalence and induces an isomorphism $K_{\#}: \pi_{1}\left(T_{f}^{T_{f}}\right.$,id $) \rightarrow$ $\pi_{1}\left(T_{\bar{f}}^{T_{\bar{f}}}, j j^{\prime}\right)$. Viewing $h$ as a path in the function space $T_{\bar{f}}^{T_{\bar{f}}}$, let $\tau_{h}: \pi_{1}\left(T_{\bar{f}}^{T_{\bar{f}}}, j j^{\prime}\right) \rightarrow$ $\pi_{1}\left(T_{\bar{f}}^{T_{\bar{f}}}\right.$,id) denote the transfer of base-point isomorphism. Letting $P_{f}$ (respectively $P_{\bar{f}}$ ) denote the rotation morphism for $f$ (respectively $\bar{f}$ ), note that $P_{\bar{f}} \circ \tau_{h} \circ K_{\#}$ equals $P_{f}$, as the trace of $h$ is constant. Hence the images of $P_{f}$ and $P_{\bar{f}}$ coincide.

Taking a cyclic homotopy $T_{f} \times I \rightarrow T_{f}$ that represents an element of $\pi_{1}\left(\left(T_{f}\right)^{T_{f}}\right.$, id $)$ and lifting it to a homotopy $\operatorname{Tel}_{f} \times I \rightarrow \operatorname{Tel}_{f}$ that begins in the identity one gets

Lemma 5.7. Let $f$ be a self-map on the connected space $Z$. There exists an element in $\pi_{1}\left(\left(T_{f}\right)^{T_{f}}\right.$, id) of rotation degree $q$ if and only if there exists an equivariant homotopy $\operatorname{Tel}_{f} \times I \rightarrow \mathrm{Tel}_{f}$ between the identity and the covering translation $\tau_{q}$.

Now we make a precise statement about what can be deduced from the proof of Theorem 6.3 of $[5$ in the noncompact case. We apply it in Corollary 5.9.

Proposition 5.8. Let $f$ be a self-map on the connected space $Z$ with $R I(f)=$ $q<\infty$. Then for any compact subset $L$ of $Z$ there exist a nonnegative number $r, a$ homotopy $N: L \times I \rightarrow Z$ between $\left.f^{r}\right|_{L}$ and $\left.f^{r+q}\right|_{L}$, and a homotopy $J: L \times I \times I \rightarrow Z$ relative to $L \times \partial I$ such that $J(z, s, 0)=f(N(z, s)), J(z, s, 1)=N(f(z), s)$ for all $z \in L$. If we assume $r$ minimal with this property, then for $L^{\prime} \supset L$ together with corresponding $r^{\prime}, N^{\prime}$ and $J^{\prime}$ we have $\left.N^{\prime}\right|_{L}=f^{r^{\prime}-r} \circ N$ and $\left.J^{\prime}\right|_{L}=f^{r^{\prime}-r} \circ J$.

Corollary 5.9. Let $f:\left(Z, z_{0}\right) \rightarrow\left(Z, z_{0}\right)$ be a self-map on the connected space $Z$ of $C W$ type. If $R I(f)$ is finite, then $f$ satisfies the property involving equation ( $\dagger)$ of Proposition 5.2. Consequently if $f$ is also a homotopy p-idempotent, $f \simeq f^{p+1}$, then its 'canonical' factorization through the telescope $\mathrm{Tel}_{f}^{+}$is splitting, and the restrictions $(\star)$ in Proposition 5.2 are isomorphisms. 
Proof. Taking $L=\left\{z_{0}\right\}$ the corresponding homotopy $N=N_{0}$ is a loop whose class in $\pi_{1}\left(Z, z_{0}\right)$ we denote by $\eta$. The homotopy $J=J_{0}$ yields $f_{\#}(\eta)=\eta$. For any $L$ the trace of $N_{L}$ is a loop of the form $f^{l} \circ N_{0}$, and its class in $\pi_{1}\left(Z, z_{0}\right)$ equals $\eta$.

Let $g:\left(S^{k}, *\right) \rightarrow\left(Z, z_{0}\right)$ represent $\gamma \in \pi_{k}\left(Z, z_{0}\right)$. Its image $\operatorname{im} g=: L$ is compact, and applying the proposition we get $r$ and the homotopies $N$ and $J$. Now $N \circ g$ is a homotopy between $f^{r} \circ g$ and $f^{r+q} \circ g$ with trace $f^{l} \circ N_{0}$. Taking classes we get $(\dagger)$ of Proposition 5.2 (note that we may always enlarge $r$ by composing with $f$ ).

The remaining assertions are immediate consequences of Proposition 5.2 .

Lemma 5.10. Let $f$ be a self-map on the connected space $Z$ with $R I(f)=q$. Then on $\mathrm{Tel}_{f}$, the iterate $\bar{f}^{q}$ is homotopic to the identity.

Proof. By Lemma 5.7 there exists an equivariant homotopy on $\mathrm{Tel}_{f}$ between id and $\tau_{q}$. Composing with $\bar{f}^{q}$ we get a homotopy between $\bar{f}^{q}$ and $\tau_{q} \circ \bar{f}^{q}$ while the latter is homotopic to the identity via the equivariant lifting $\bar{R}_{q}$ of the $q$-th tumble.

Lemma 5.11. Let $f$ be a self-map on the connected space $Z$. If $R I(f)$ is finite, then so is $R I\left(f^{r}\right)$, for all $r$, and $R I\left(f^{r}\right) \leqslant L C M(r, R I(f)) / r$.

Proof. Let $l=\operatorname{LCM}(r, q)$ be the least common multiple of $r$ and $q$ and set $q^{\prime}=l / r$. If $R I(f)=q$, there exist an element of $\pi_{1}\left(\left(T_{f}\right)^{T_{f}}\right.$, id) of rotation degree $l$ and, by Lemma 5.7, an equivariant homotopy $\bar{F}: \operatorname{Tel}_{f} \times I \rightarrow \mathrm{Tel}_{f}$ between the identity and $\tau_{l}$. The relations $\alpha \tau_{1}=\tau_{r} \alpha$ and $\beta \tau_{r}=\tau_{1} \beta$ render $\beta \circ \bar{F}\left(\alpha\left({ }_{-}\right)\right.$, $)$an equivariant homotopy between $\beta \alpha$ and $\beta \tau_{l} \alpha=\tau_{q^{\prime}} \beta \alpha$. As $\beta \alpha$ is equivariantly homotopic to the identity, this accounts for an equivariant homotopy between the identity and $\tau_{q^{\prime}}$ on $\mathrm{Tel}_{f^{r}}$. A reapplication of Lemma 5.7 yields $R I\left(f^{r}\right)$ finite and a divisor of $q^{\prime}$.

Proof of Theorem 1.1 and Corollary 1.2, Let $f^{r} \simeq f^{r+p}$. Then $f^{r}$ is a homotopy $p$-idempotent. If $f^{r}$ splits, then on homotopy groups, $\operatorname{im} f_{*}^{r}=\operatorname{im} f_{*}^{r+1}=\cdots$, by Theorems 2.3 and 2.1. We can use this in place of $(\dagger)$ in the proof of Proposition 5.2 to infer that for $d_{r}: Z \rightarrow T e l_{f^{r}}$ the restrictions $\left.d_{r *}\right|_{i m f_{*}^{r}}: \operatorname{im} f_{*}^{r} \rightarrow \pi_{k}\left(\operatorname{Tel}_{f^{r}}\right)$ are isomorphisms. The composite $Z \stackrel{d}{\rightarrow} \operatorname{Tel}_{f} \stackrel{\beta}{\rightarrow} \operatorname{Tel}_{f^{r}}$ equals $Z \stackrel{d_{r}}{\rightarrow} \mathrm{Tel}_{f^{r}}$, and as $\beta$ is a homotopy equivalence, the restrictions $\left.d_{*}\right|_{\operatorname{im} f_{*}^{r}}: \operatorname{im} f_{*}^{r} \rightarrow \pi_{k}\left(\operatorname{Tel}_{f}\right)$ are isomorphisms as well. Lemma 5.6 now yields the equality $R I(f)=R I(\bar{f})$, where $\bar{f}: \operatorname{Tel}_{f} \rightarrow \operatorname{Tel}_{f}$ is the induced map on the telescope of $f$.

Corollary 2.4, (i), and Theorem 2.1 imply that $f^{r p}$ is a split idempotent and thus by Proposition 3.1 of Geoghegan and Nicas [6], $R I\left(f^{r p}\right)=1$. By Lemma 5.10, the map $\overline{f^{r p}}$ : $\operatorname{Tel}_{f^{r p}} \rightarrow \operatorname{Tel}_{f^{r p}}$ is homotopic to the identity. But $\overline{f^{r p}}=\bar{f}^{r p}$, where $\bar{f}$ is the induced self-map on $\operatorname{Tel}_{f^{r p}}$. As $\operatorname{Tel}_{f} \stackrel{\bar{f}}{\rightarrow} \operatorname{Tel}_{f} \stackrel{\beta}{\rightarrow} \operatorname{Tel}_{f^{r p}}$ equals $\operatorname{Tel}_{f} \stackrel{\beta}{\rightarrow} \operatorname{Tel}_{f^{r p}} \stackrel{\bar{f}}{\rightarrow} \operatorname{Tel}_{f^{r p}}$, the iterate $\bar{f}^{r p}$ on $\mathrm{Tel}_{f}$ is homotopic to the identity.

A conjunction of Theorem 2.1 and the equivalence $(\mathbf{i}) \Longleftrightarrow$ (ii) of Theorem 2.3 also renders $f^{r+1}$ a split $p$-idempotent. Repeating the above argument we infer that on $\mathrm{Tel}_{f}$, the iterate $\bar{f}^{(r+1) p}$ is homotopic to the identity.

Therefore $\bar{f}^{p}$ is homotopic to the identity on $\mathrm{Tel}_{f}$. By Theorem 2.4 of Geoghegan and Nicas [], $R I(\bar{f})$ is finite and divides $p^{2}$. As $R I(f)=R I(\bar{f})$ we are done.

Conversely, assume that $R I(f)$ is finite. Then by Lemma [5.11 $R I\left(f^{r}\right)$ is also finite, rendering $f^{r}$ a split $p$-idempotent by Corollary 5.9. By what we have shown above it follows that $R I(f)$ divides $p^{2}$.

The theorem, Lemma 5.11. and Theorems 2.3 and 2.1 imply the corollary. 


\section{REFERENCES}

[1] Matthew G. Brin and Fernando Guzmán, Automorphisms of generalized Thompson groups. J. Algebra 203 (1998), no. 1, 285-348. MR1620674 (99d:20056)

[2] K. S. Brown, Finiteness properties of groups. J. Pure Appl. Algebra 44 (1987), no. 1-3, 45-75. MR885095 (88m:20110)

[3] Kenneth S. Brown and Ross Geoghegan, An infinite-dimensional torsion-free $\mathrm{FP}_{\infty}$ group. Invent. Math. 77 (1984), no. 2, 367-381. MR752825 (85m:20073)

[4] Peter J. Freyd and Alex Heller, Splitting homotopy idempotents. II. J. Pure Appl. Algebra 89 (1993), no. 1-2, 93-106. MR.1239554 (95h:55015)

[5] Ross Geoghegan and Andrew Nicas, Higher Euler characteristics. I. Enseign. Math. (2) 41 (1995), no. 1-2, 3-62. MR1341940 (96f:55002)

[6] Ross Geoghegan and Andrew Nicas, Homotopy periodicity and coherence. Proc. Amer. Math. Soc. 124 (1996), no. 9, 2889-2895. MR.1346975 (96k:55011)

[7] Harold M. Hastings and Alex Heller, Homotopy idempotents on finite-dimensional complexes split.

Fakulteta za matematiko in fiziko, Jadranska ulica 19, SI-1111 Ljubluana, Slovenia

E-mail address: jaka.smrekar@fmf.uni-lj.si 